\title{
The stationary Weyl equation and Cosserat elasticity
}

\author{
Olga Chervova* \\ Institute of Origins, University College London, Gower Street, London WC1E 6BT, UK \\ Dmitri Vassiliev \\ Department of Mathematics and Institute of Origins, \\ University College London, Gower Street, London WC1E 6BT, UK
}

(Dated: December 2, 2018)

\begin{abstract}
The paper deals with the Weyl equation which is the massless Dirac equation. We study the Weyl equation in the stationary setting, i.e. when the spinor field oscillates harmonically in time. We suggest a new geometric interpretation of the stationary Weyl equation, one which does not require the use of spinors, Pauli matrices or covariant differentiation. We think of our 3-dimensional space as an elastic continuum and assume that material points of this continuum can experience no displacements, only rotations. This framework is a special case of the Cosserat theory of elasticity. Rotations of material points of the space continuum are described mathematically by attaching to each geometric point an orthonormal basis which gives a field of orthonormal bases called the coframe. As the dynamical variables (unknowns) of our theory we choose the coframe and a density. We choose a particular potential energy which is conformally invariant and then incorporate time into our action in the standard Newtonian way, by subtracting kinetic energy. The main result of our paper is the theorem stating that in the stationary setting our model is equivalent to a pair of Weyl equations. The crucial element of the proof is the observation that our Lagrangian admits a factorization.
\end{abstract}

PACS numbers: 11.10.Lm, 14.60.Lm, 46.05.+b

Keywords: neutrino, spin, torsion, Cosserat elasticity

\section{MAIN RESULT}

Throughout this paper we work on a 3-manifold $M$ equipped with local coordinates $x^{\alpha}, \alpha=1,2,3$, and prescribed positive metric $g_{\alpha \beta}$ which does not depend on time. We extend the Riemannian 3-manifold $M$ to a Lorentzian 4-manifold $\mathbb{R} \times M$ by adding the time coordinate $x^{0} \in \mathbb{R}$. The metric on $\mathbb{R} \times M$ is defined as

$$
\mathbf{g}_{\boldsymbol{\alpha} \boldsymbol{\beta}}=\left(\begin{array}{cc}
-1 & 0 \\
0 & g_{\alpha \beta}
\end{array}\right) \text {. }
$$

Here and further on we use bold type for extended quantities. Say, the use of bold type in tensor indices $\boldsymbol{\alpha}, \boldsymbol{\beta}$ appearing in the LHS of formula (11) indicates that these run through the values $0,1,2,3$, whereas the use of normal type in tensor indices $\alpha, \beta$ appearing in the RHS of formula (1) indicates that these run through the values $1,2,3$.

All constructions presented in the paper are local so we do not make a priori assumptions on the geometric structure of $\{M, g\}$.

The Weyl equation is the massless Dirac equation. It is the accepted mathematical model for a massless neutrino field. The dynamical variable (unknown quantity) in the Weyl equation is a two-component complex-valued spinor field $\xi$ which is a function of time $x^{0} \in \mathbb{R}$ and local coordinates $x^{\alpha}$ on $M$. The explicit form of the Weyl equation is

$$
i\left( \pm \sigma_{\dot{a} b}^{0} \partial_{0}+\sigma^{\alpha}{ }_{\dot{a} b} \nabla_{\alpha}\right) \xi^{b}=0 .
$$

Here the $\sigma$ are Pauli matrices, $\partial_{0}$ is the time derivative and $\nabla_{\alpha}$ is the covariant spatial derivative, see Appendix B for details. Summation in (2) is carried out over the tensor index $\alpha=1,2,3$ as well as over the spinor in$\operatorname{dex} b=1,2$. The use of the partial derivative $\partial_{0}=\partial / \partial x^{0}$ in equation (2) is justified by the fact that the time coordinate $x^{0}$ is fixed and we allow only changes of coordinates $\left(x^{1}, x^{2}, x^{3}\right)$ which do not depend on $x^{0}$.

We see that the Weyl equation (2) is a system of two $(\dot{a}=\dot{1}, \dot{2})$ complex linear partial differential equations on the 4-manifold $\mathbb{R} \times M$ for two complex unknowns $\xi^{b}$, $b=1,2$. The two choices of sign in (2) give two versions of the Weyl equation which differ by time reversal. Thus, we have a pair of Weyl equations.

We will be interested in spinor fields of the form

$$
\xi\left(x^{0}, x^{1}, x^{2}, x^{3}\right)=e^{-i p_{0} x^{0}} \eta\left(x^{1}, x^{2}, x^{3}\right)
$$

where

$$
p_{0} \neq 0
$$

is a real number. Substituting (3) into (2) we get the equation

$$
\pm p_{0} \sigma_{\dot{a} b}^{0} \eta^{b}+i \sigma^{\alpha}{ }_{\dot{a} b} \nabla_{\alpha} \eta^{b}=0
$$

which we shall call the stationary Weyl equation. The difference between equations (2) and (5) is that in equation (2) the spinor field $\xi$ "lives" on the Lorentzian 4- 
$\eta$ "lives" on the Riemannian 3-manifold $M$. Thus, the stationary Weyl equation is the Weyl equation with time separated out. The relationship between equations (2) and (5) is the same as between the wave equation and the Helmholtz equation.

The stationary Weyl equation (5) is the object of study of this paper. We separated out time to simplify the problem while retaining most of its essential features. Note also that this separation of variables has a clear physical meaning: the real number $p_{0}$ appearing in (3) and (5) is quantum mechanical energy.

The aim of our paper is to show that the stationary Weyl equation (5) can be reformulated in an alternative (but mathematically equivalent) way using instead of a spinor field a different set of dynamical variables. Namely, we view our 3-manifold $M$ as an elastic continuum whose material points can experience no displacements, only rotations, with rotations of different material points being totally independent. The idea of rotating material points may seem exotic, however it has long been accepted in continuum mechanics within the Cosserat theory of elasticity [1]. This idea also lies at the heart of the theory of teleparallelism (= absolute parallelism $=$ fernparallelismus), a subject promoted by A. Einstein and É. Cartan [2-4]. See Section $[\mathrm{IX}$ for more details.

Rotations of material points of the 3-dimensional elastic continuum are described mathematically by attaching to each geometric point of the manifold $M$ an orthonormal basis, which gives a field of orthonormal bases called the frame or coframe, depending on whether one prefers dealing with vectors or covectors. Our mathematical model will be built on the basis of exterior calculus (no need for covariant derivatives) so for us it will be more natural to use the coframe.

The coframe $\vartheta$ is a triple of orthonormal covector fields $\vartheta^{j}, j=1,2,3$, on the 3-manifold $M$. Each covector field $\vartheta^{j}$ can be written more explicitly as $\vartheta^{j}{ }_{\alpha}$ where the tensor index $\alpha=1,2,3$ enumerates the components. The orthonormality condition for the coframe can be represented as a single tensor identity

$$
g=\delta_{j k} \vartheta^{j} \otimes \vartheta^{k}
$$

where $\delta_{j k}$ is the Kronecker delta. For the sake of clarity we repeat formula (6) giving tensor indices explicitly and performing summation over frame indices explicitly:

$$
g_{\alpha \beta}=\delta_{j k} \vartheta^{j}{ }_{\alpha} \vartheta^{k}{ }_{\beta}=\vartheta^{1}{ }_{\alpha} \vartheta^{1}{ }_{\beta}+\vartheta_{\alpha}^{2} \vartheta^{2}{ }_{\beta}+\vartheta_{\alpha}^{3} \vartheta_{\beta}^{3}
$$

where $\alpha$ and $\beta$ run through the values $1,2,3$. We view the identity (6) as a kinematic constraint: the metric $g$ is given (prescribed) and the coframe elements $\vartheta^{j}$ are chosen so that they satisfy (6), which leaves us with three real degrees of freedom at every point of $M$.

As dynamical variables in our model we choose the coframe $\vartheta$ and a positive density $\rho$. Our coframe and density are functions of local coordinates $\left(x^{1}, x^{2}, x^{3}\right)$ on $M$ as well as of time $x^{0}$.
At a physical level, making the density $\rho$ a dynamical variable means that we view our continuum more like a fluid rather than a solid. In other words, we allow the material to redistribute itself so that it finds its equilibrium distribution.

Note that the total number of real dynamical degrees of freedom contained in the coframe $\vartheta$ and positive density $\rho$ is four, exactly as in a two-component complex-valued spinor field $\xi$. Moreover, it is known (see Appendix C) that a coframe $\vartheta$ and a (positive) density $\rho$ are geometrically equivalent to a nonvanishing spinor field $\xi$ modulo the sign of $\xi$.

The crucial element in our construction is the choice of potential energy. It is known that in the purely rotational setting the potential energy of a physically linear elastic continuum contains three quadratic terms, with three real parameters (elastic moduli) as factors. The three quadratic terms in potential energy correspond to the three irreducible pieces of torsion. It is not a priori clear what the elastic moduli of "world aether" are. We choose a potential energy which feels only one piece of torsion, axial, and is, moreover, conformally invariant, i.e. which is invariant under the rescaling of the 3-dimensional metric $g$ by an arbitrary positive scalar function. This leaves us with a unique, up to rescaling by a positive constant, formula (12) for potential energy.

After the potential energy is chosen the remainder of our construction is straightforward. We incorporate time into our action in the standard Newtonian way, by subtracting kinetic energy. This gives us the Lagrangian density (19). As we are interested in comparing our mathematical model with the Weyl equation, we perform a change of dynamical variables and switch from coframe $\vartheta$ and density $\rho$ to a spinor field $\xi$. Our Lagrangian density now takes the form (20). We write down the field equation (Euler-Lagrange equation) for our Lagrangian density and observe that time separates out if we seek stationary solutions (3); this separation of variables is highly nontrivial because our field equation is nonlinear. After separation of variables our Lagrangian density takes the stationary form (28).

The main result of our paper is the following

Theorem 1 A nonvanishing time-independent spinor field $\eta$ is a solution of the field equation for our stationary Lagrangian density (28) if and only if it is a solution of one of the two stationary Weyl equations (5).

Theorem 1 provides an elementary, in terms of Newtonian mechanics and elasticity theory, interpretation of the stationary Weyl equation. This interpretation is geometrically much simpler than the traditional one as the mathematical description of our model does not require the use of spinors, Pauli matrices or covariant differentiation.

The only technical assumption contained in the statement of Theorem 1 and its proof is that the density does not vanish which is equivalent to the spinor field not vanishing. At the moment we do not know how to drop this 
technical assumption. We can only remark that generically one would not expect a spinor field $\eta$ "living" on a 3 -manifold to vanish as this would mean satisfying four real equations $\operatorname{Re} \eta^{1}=\operatorname{Im} \eta^{1}=\operatorname{Re} \eta^{2}=\operatorname{Im} \eta^{2}=0$ having at our disposal only three real variables $x^{\alpha}, \alpha=1,2,3$.

The crucial element of the proof of Theorem 1 is the observation that our Lagrangian density admits factorization, see formula (31). Thus, our argument is similar to the original argument of Dirac, the difference being that we factorize the Lagrangian whereas Dirac factorized the field equation (Klein-Gordon equation). In our model factorizing the field equation is impossible because the equation is nonlinear.

Our paper has the following structure. In Section II we describe our mathematical model, in Section [II we switch to the language of spinors, in Section IV we separate out time and in Section $\nabla$ we factorize our Lagrangian. Section VI contains the proof of Theorem 1 . In Section VII we analyze plane wave solutions and in Section VIII we give a relativistic representation of our Lagrangian. The concluding discussion is presented in Section [X].

Our paper also has five appendices dealing with issues of a more technical nature: Appendix $\mathrm{A}$ describes our general notation, Appendix $\mathrm{B}$ describes our spinor notation, Appendix $\mathrm{C}$ gives the correspondence between coframes and spinors, Appendix D gives the spinor representation of axial torsion and angular velocity and Appendix E deals with a toy model which illustrates how a particular class of nonlinear second order differential equations reduces to pairs of linear first order equations.

\section{OUR MODEL}

In this section we describe in detail our mathematical model. At a basic level it was already sketched out in Section [.

We need to write down the potential energy of a deformed Cosserat continuum. The natural measure of deformations caused by rotations of material points is the torsion tensor defined by the explicit formula

$$
T:=\delta_{j k} \vartheta^{j} \otimes d \vartheta^{k}
$$

where $d$ denotes the exterior derivative. Here "torsion" means "torsion of the teleparallel connection" with "teleparallel connection" defined by the condition that the covariant derivative of each coframe element $\vartheta^{j}$ is zero; see Appendix A of [5] for a concise exposition.

Our construction of potential energy follows the logic of classical linear elasticity [6], the only difference being that instead of a rank 2 tensor (strain) we deal with a rank 3 tensor (torsion). The logic of classical linear elasticity dictates that we must first decompose our measure of deformation (torsion) into irreducible pieces, with irreducibility understood in terms of invariance under changes of local coordinates preserving the metric $g_{\alpha \beta}$ at a given point $P \in M$. It is known [7] that torsion has three irreducible pieces labeled by the adjectives axial, vector and tensor. (Vector torsion is sometimes called trace torsion.) The general formula for the potential energy of a homogeneous isotropic linear elastic material contains squares of all irreducible pieces with some constant coefficients in front. Thus, the general formula for potential energy should contain three free parameters (elastic moduli).

We, however, choose to construct our potential energy using only one piece of torsion, namely, the axial piece given by the explicit formula

$$
T^{\mathrm{ax}}:=\frac{1}{3} \delta_{j k} \vartheta^{j} \wedge d \vartheta^{k} .
$$

Comparing (8) with (7) we see that axial torsion has a very simple meaning: it is the totally antisymmetric part of the torsion tensor ( $T$ is antisymmetric only in the last pair of indices whereas $T^{\mathrm{ax}}$ is antisymmetric in all three). In other words, $T^{\text {ax }}$ is a 3 -form.

We chose the axial piece of torsion because it has two remarkable properties.

- The definition of axial torsion (8) is very simple in that it does not involve the metric. In a sense, axial torsion (3-form) is an analogue of the electromagnetic field tensor (2-form) from Maxwell's theory.

- Axial torsion possesses the property of conformal covariance, i.e. scales nicely under conformal rescalings of the metric. Indeed, it is easy to see that if we rescale our coframe as

$$
\vartheta^{j} \mapsto e^{h} \vartheta^{j}
$$

where $h: M \rightarrow \mathbb{R}$ is an arbitrary scalar function, then our metric scales as

$$
g_{\alpha \beta} \mapsto e^{2 h} g_{\alpha \beta}
$$

and axial torsion scales as

$$
T^{\mathrm{ax}} \mapsto e^{2 h} T^{\mathrm{ax}}
$$

without the derivatives of $h$ appearing. The fact that axial torsion is conformally covariant was previously observed by Yu. N. Obukhov [8] and J. M. Nester [9].

We take the potential energy of our continuum to be

$$
P\left(x^{0}\right):=\int_{M}\left\|T^{\mathrm{ax}}\right\|^{2} \rho d x^{1} d x^{2} d x^{3} .
$$

It is easy to see that the potential energy (12) is conformally invariant: it does not change if we rescale our coframe as (91) and our density as

$$
\rho \mapsto e^{2 h} \rho .
$$

This follows from formulas (11), (10) and

$$
\left\|T^{\mathrm{ax}}\right\|^{2}=\frac{1}{3 !} T_{\alpha \beta \gamma}^{\mathrm{ax}} T_{\kappa \lambda \mu}^{\mathrm{ax}} g^{\alpha \kappa} g^{\beta \lambda} g^{\gamma \mu} .
$$


We take the kinetic energy of our continuum to be

$$
K\left(x^{0}\right):=\int_{M}\|\dot{\vartheta}\|^{2} \rho d x^{1} d x^{2} d x^{3}
$$

where $\dot{\vartheta}$ is the 2 -form

$$
\dot{\vartheta}:=\frac{1}{3} \delta_{j k} \vartheta^{j} \wedge \partial_{0} \vartheta^{k}
$$

(compare with (8)). The 2-form (15) can, of course, be written as

$$
\dot{\vartheta}=\frac{2}{3} * \omega
$$

where

$$
\omega:=\frac{1}{2} *\left(\delta_{j k} \vartheta^{j} \wedge \partial_{0} \vartheta^{k}\right)
$$

is the (pseudo)vector of angular velocity. Hence, (14) is the standard expression for the kinetic energy of a homogeneous isotropic Cosserat continuum. In writing formula (14) we assumed homogeneity (properties of the material are the same at all points of the manifold $M$ ) and isotropy (properties of the material are invariant under rotations of the local coordinate system). We think of each material point as a uniform ball possessing a moment of inertia and without a preferred axis of rotation.

We now combine the potential energy (12) and kinetic energy (14) to form the action (variational functional) of our dynamic problem:

$$
\begin{aligned}
S(\vartheta, \rho):=\int_{\mathbb{R}}\left(P\left(x^{0}\right)-K\left(x^{0}\right)\right) d x^{0} & \\
= & \int_{\mathbb{R} \times M} L(\vartheta, \rho) d x^{0} d x^{1} d x^{2} d x^{3}
\end{aligned}
$$

where

$$
L(\vartheta, \rho):=\left(\left\|T^{\mathrm{ax}}\right\|^{2}-\|\dot{\vartheta}\|^{2}\right) \rho
$$

is our Lagrangian density. Note that our construction of the action (18) out of potential and kinetic energies is Newtonian (compare with classical elasticity or even the harmonic oscillator in classical mechanics).

Our field equations (Euler-Lagrange equations) are obtained by varying the action (18) with respect to the coframe $\vartheta$ and density $\rho$. Varying with respect to the density $\rho$ is easy: this gives the field equation $\left\|T^{\mathrm{ax}}\right\|^{2}=\|\dot{\vartheta}\|^{2}$ which is equivalent to $L(\vartheta, \rho)=0$. Varying with respect to the coframe $\vartheta$ is more difficult because we have to maintain the kinematic constraint (6); recall that the metric is assumed to be prescribed (fixed).

A technique for varying the coframe with kinematic constraint (6) was described in Appendix B of [5]. We, however, do not write down the field equations for the Lagrangian density (19) explicitly. We note only that they are highly nonlinear and do not appear to bear any resemblance to the linear Weyl equation (2).
Remark 1 The 3-form $T^{\mathrm{ax}}$ and 2-form $\dot{\vartheta}$ are invariant under rigid rotations of the coframe, i.e. under special orthogonal transformations (A3) with constant $O^{j}{ }_{k}$. Hence, our Lagrangian density (19) is invariant under rigid rotations of the coframe and, accordingly, solutions of our field equations whose coframes differ by a rigid rotation can be collected into equivalence classes. Further on we view coframes differing by a rigid rotation as equivalent.

\section{SWITCHING TO THE LANGUAGE OF SPINORS}

As pointed out in the previous section, varying the coframe subject to the kinematic constraint (6) is not an easy task. This technical difficulty can be overcome by switching to a different dynamical variable. Namely, it is known, see Appendix C, that in dimension 3 a coframe $\vartheta$ and a (positive) density $\rho$ are equivalent to a nonvanishing spinor field $\xi$ modulo the sign of $\xi$. The great advantage of switching to a spinor field $\xi$ is that there are no kinematic constraints on its components, so the derivation of field equations becomes absolutely straightforward.

We now need to substitute formulas (C1), (C3) and (C4) into (8) and (15) to get explicit expressions for $T^{\mathrm{ax}}$ and $\dot{\vartheta}$ in terms of the spinor field $\xi$. The results are presented in Appendix [D] Namely, formula (D1) gives the spinor representation of the 3 -form $T^{\text {ax }}$ whereas formulas (D2) and (16) give the spinor representation of the 2 -form $\dot{\vartheta}$. We also know the spinor representation for our density $\rho$, see formulas (C1) and (C2). Substituting all these into formula (19) we arrive at the following self-contained explicit spinor representation of our Lagrangian density

$$
\begin{aligned}
L(\xi) & =\frac{4}{9 \bar{\xi}^{\dot{c}} \sigma_{0 \dot{c} d} \xi^{d}}\left(\left[i\left(\bar{\xi}^{\dot{a}} \sigma^{\alpha}{ }_{\dot{a} b} \nabla_{\alpha} \xi^{b}-\xi^{b} \sigma^{\alpha}{ }_{\dot{a} b} \nabla_{\alpha} \bar{\xi}^{\dot{a}}\right)\right]^{2}\right. \\
& \left.-\left\|i\left(\bar{\xi}^{\dot{a}} \sigma_{\alpha \dot{a} b} \partial_{0} \xi^{b}-\xi^{b} \sigma_{\alpha \dot{a} b} \partial_{0} \bar{\xi}^{\dot{a}}\right)\right\|^{2}\right) \sqrt{\operatorname{det} g}
\end{aligned}
$$

Here and further on we write our Lagrangian density and our action as $L(\xi)$ and $S(\xi)$ rather than $L(\vartheta, \rho)$ and $S(\vartheta, \rho)$, thus indicating that we have switched to spinors. The nonvanishing spinor field $\xi$ is the new dynamical variable and it will be varied without any constraints.

Straightforward calculations show that the field equation for our Lagrangian density (20) is

$$
\begin{array}{r}
-\frac{4 i}{3}\left(\left(* T^{\mathrm{ax}}\right) \sigma^{\alpha}{ }_{\dot{a} b} \nabla_{\alpha} \xi^{b}+\sigma^{\alpha}{ }_{\dot{a} b} \nabla_{\alpha}\left(\left(* T^{\mathrm{ax}}\right) \xi^{b}\right)\right) \\
-\frac{8 i}{9}\left(\omega_{\alpha} \sigma^{\alpha}{ }_{\dot{a} b} \partial_{0} \xi^{b}+\sigma^{\alpha}{ }_{\dot{a} b} \partial_{0}\left(\omega_{\alpha} \xi^{b}\right)\right) \\
-\rho^{-1} L \sigma_{0 \dot{a} b} \xi^{b}=0
\end{array}
$$

where the geometric quantities $* T^{\mathrm{ax}}, \omega, \rho$ and $L$ are expressed via the spinor field $\xi$ in accordance with formu- 
las (D1), (D2), (C1), (C2) and (20). The LHS of equation (21) is the spinor field $F_{\dot{a}}$ appearing in the formula for the variation of the action (18):

$$
\delta S=\int_{\mathbb{R} \times M}\left(F_{\dot{a}} \delta \bar{\xi}^{\dot{a}}+\bar{F}_{a} \delta \xi^{a}\right) \sqrt{\operatorname{det} g} d x^{0} d x^{1} d x^{2} d x^{3} .
$$

We shall refer to equation (21) as the dynamic field equation, with "dynamic" indicating that it contains the time derivative $\partial_{0}$.

\section{SEPARATING OUT TIME}

Our dynamic field equation (21) is highly nonlinear and one does expect it to admit separation of variables. Nevertheless, we seek solutions of the form (3). Substituting formula (3) into formulas (D1), (D2), (C1), (C2) and (20) and using the identity (B5) we get

$$
\begin{gathered}
* T^{\mathrm{ax}}=-\frac{2 i\left(\bar{\eta}^{\dot{a}} \sigma^{\alpha}{ }_{\dot{a} b} \nabla_{\alpha} \eta^{b}-\eta^{b} \sigma^{\alpha}{ }_{\dot{a} b} \nabla_{\alpha} \bar{\eta}^{\dot{a}}\right)}{3 \bar{\eta}^{\dot{c}} \sigma_{0 \dot{c} d} \eta^{d}} \\
\omega_{\alpha}=\frac{2 p_{0} \bar{\eta}^{\dot{a}} \sigma_{\alpha \dot{a} b} \eta^{b}}{\bar{\eta}^{\dot{c}} \sigma_{0 \dot{c} d} \eta^{d}} \\
\rho=\bar{\eta}^{\dot{a}} \sigma_{0 \dot{a} b} \eta^{b} \sqrt{\operatorname{det} g} \\
L(\eta)=\frac{16}{9 \bar{\eta}^{\dot{c}} \sigma_{0 \dot{c} d} \eta^{d}}\left(\left[\frac{i}{2}\left(\bar{\eta}^{\dot{a}} \sigma^{\alpha}{ }_{\dot{a} b} \nabla_{\alpha} \eta^{b}-\eta^{b} \sigma^{\alpha}{ }_{\dot{a} b} \nabla_{\alpha} \bar{\eta}^{\dot{a}}\right)\right]^{2}\right. \\
\left.-\left(p_{0} \bar{\eta}^{\dot{a}} \sigma_{0 \dot{a} b} \eta^{b}\right)^{2}\right) \sqrt{\operatorname{det} g}
\end{gathered}
$$

Note that the geometric quantities (22)-(25) do not depend on time $x^{0}$, which simplifies the next step: substituting (3) into our dynamic field equation (21), using the identity (B5) and dividing through by the common factor $e^{-i p_{0} x^{0}}$ we get

$$
\begin{aligned}
&-\frac{4 i}{3}\left(\left(* T^{\mathrm{ax}}\right) \sigma^{\alpha}{ }_{\dot{a} b} \nabla_{\alpha} \eta^{b}+\sigma^{\alpha}{ }_{a b} \nabla_{\alpha}\left(\left(* T^{\mathrm{ax}}\right) \eta^{b}\right)\right) \\
&-\frac{32 p_{0}^{2}}{9} \sigma_{0 \dot{a} b} \eta^{b}-\rho^{-1} L \sigma_{0 \dot{a} b} \eta^{b}=0 .
\end{aligned}
$$

The remarkable feature of formulas (22)-(26) is that they do not contain dependence on time $x^{0}$. Thus, we have shown that our dynamic field equation (21) admits separation of variables, i.e. one can seek solutions in the form (3).

We shall refer to equation (26) as the stationary field equation, with "stationary" indicating that time $x^{0}$ has been separated out.

Consider now the action

$$
S(\eta):=\int_{M} L(\eta) d x^{1} d x^{2} d x^{3}
$$

where $L(\eta)$ is our "stationary" Lagrangian density (25). It is easy to see that our stationary field equation (26) is the Euler-Lagrange equation for our "stationary" action (27).

In the remainder of the paper we do not use the explicit form of the stationary field equation (26), dealing only with the stationary Lagrangian density (25) and the stationary action (27). We needed the explicit form of field equations, dynamic and stationary, only to justify separation of variables.

It appears that the underlying group-theoretic reason for our nonlinear dynamic field equation (21) admitting separation of variables is the fact that our model is $\mathrm{U}(1)$ invariant, i.e. it is invariant under the multiplication of the spinor field $\xi$ by a complex constant of modulus 1 . Hence, it is feasible that one could have performed the separation of variables argument without even writing down the explicit form of field equations.

We give for reference a more compact representation of our stationary Lagrangian density (25) in terms of axial torsion $T^{\mathrm{ax}}$ (see formula (22) ) and density $\rho$ (see formula (24)):

$$
L(\eta)=\left(\left\|T^{\mathrm{ax}}\right\|^{2}-\frac{16}{9} p_{0}^{2}\right) \rho .
$$

Of course, formula (28) is our original formula (19) with time separated out. The choice of dynamical variables in the stationary Lagrangian density (28) is up to the user: one can either use the time-independent spinor field $\eta$ or, equivalently, the corresponding timeindependent coframe and time-independent density (the latter are related to $\eta$ by formulas (C1)-(C4) with $\xi$ replaced by $\eta$ ). The important thing is that now our dynamical variables are time-independent because we have separated out time.

The fact that we use the same notation $L$ both for the dynamic and stationary Lagrangian densities should not cause problems as in all subsequent sections, apart form Section VIII, we deal with the stationary case only.

\section{FACTORIZATION OF OUR LAGRANGIAN}

Put

$$
\begin{array}{r}
L_{ \pm}(\eta):=\left[\frac{i}{2}\left(\bar{\eta}^{\dot{a}} \sigma^{\alpha}{ }_{a b} \nabla_{\alpha} \eta^{b}-\eta^{b} \sigma^{\alpha}{ }_{a} \nabla_{\alpha} \bar{\eta}^{\dot{a}}\right)\right. \\
\left. \pm p_{0} \bar{\eta}^{\dot{a}} \sigma^{0}{ }_{a} b \eta^{b}\right] \sqrt{\operatorname{det} g} .
\end{array}
$$

This is the Lagrangian density for the stationary Weyl equation (5). Formula (29) can be written in more compact form as

$$
L_{ \pm}(\eta)=\left(-\frac{3}{4} * T^{\mathrm{ax}} \mp p_{0}\right) \rho
$$

where $* T^{\mathrm{ax}}$ is the Hodge dual of axial torsion, see formula (22), and $\rho$ is the density, see formula (24). Com- 
paring formulas (28) and (30) we get

$$
L(\eta)=-\frac{32 p_{0}}{9} \frac{L_{+}(\eta) L_{-}(\eta)}{L_{+}(\eta)-L_{-}(\eta)} .
$$

Let us emphasize once again that throughout this paper we assume that the density $\rho$ does not vanish, which is, of course, equivalent to the spinor field not vanishing. In view of formulas (30) and (44) in the stationary case the assumption $\rho \neq 0$ can be equivalently rewritten as

$$
L_{+}(\eta) \neq L_{-}(\eta)
$$

so the denominator in (31) is nonzero.

Formula (31) is the centerpiece of our paper: it establishes the connection between Cosserat elasticity and the Weyl equation. Moreover, the fact that the RHS of formula (31) contains a product of two Weyl Lagrangian densities shows that we are essentially following Dirac's factorization construction, the difference being that in the nonlinear setting we cannot factorize equations and have to settle for the next best thing - factorizing the Lagrangian.

\section{PROOF OF THEOREM 1}

Observe that the Lagrangian densities $L_{ \pm}$defined by formula (29) possess the property of scaling covariance:

$$
L_{ \pm}\left(e^{h} \eta\right)=e^{2 h} L_{ \pm}(\eta)
$$

where $h: M \rightarrow \mathbb{R}$ is an arbitrary scalar function. In fact, the Lagrangian density of any formally selfadjoint (symmetric) linear first order partial differential operator has the scaling covariance property (33).

We claim that the statement of Theorem 1 follows from formulas (31) and (33). The proof presented below is an abstract one and does not depend on the physical nature of the dynamical variable $\eta$, the only requirement being that it is an element of a vector space so that scaling makes sense.

Note that formulas (31) and (33) imply that the Lagrangian density $L$ possesses the property of scaling covariance, so all three of our Lagrangian densities, $L, L_{+}$ and $L_{-}$, have this property. Note also that if $\eta$ is a solution of the field equation for some Lagrangian density $\mathcal{L}$ possessing the property of scaling covariance then $\mathcal{L}(\eta)=0$. Indeed, let us perform a scaling variation of our dynamical variable

$$
\eta \mapsto \eta+h \eta
$$

where $h: M \rightarrow \mathbb{R}$ is an arbitrary "small" scalar function with compact support. Then $0=\delta \int \mathcal{L}(\eta)=2 \int h \mathcal{L}(\eta)$ which holds for arbitrary $h$ only if $\mathcal{L}(\eta)=0$.

In the remainder of the proof the variations of $\eta$ are arbitrary and not necessarily of the scaling type (34).

Suppose that $\eta$ is a solution of the field equation for the Lagrangian density $L_{+}$. [The case when $\eta$ is a solution of the field equation for the Lagrangian density $L_{-}$ is handled similarly.] Then $L_{+}(\eta)=0$ and, in view of formula (32), $L_{-}(\eta) \neq 0$. Varying $\eta$ we get

$$
\begin{aligned}
& \delta \int L(\eta)=-\frac{32 p_{0}}{9}\left(\int \frac{L_{-}(\eta)}{L_{+}(\eta)-L_{-}(\eta)} \delta L_{+}(\eta)\right. \\
&\left.+\int L_{+}(\eta) \delta \frac{L_{-}(\eta)}{L_{+}(\eta)-L_{-}(\eta)}\right)= \frac{32 p_{0}}{9} \int \delta L_{+}(\eta) \\
&=\frac{32 p_{0}}{9} \delta \int L_{+}(\eta)
\end{aligned}
$$

So

$$
\delta \int L(\eta)=\frac{32 p_{0}}{9} \delta \int L_{+}(\eta) .
$$

We assumed that $\eta$ is a solution of the field equation for the Lagrangian density $L_{+}$so $\delta \int L_{+}(\eta)=0$ and formula (35) implies that $\delta \int L(\eta)=0$. As the latter is true for an arbitrary variation of $\eta$ this means that $\eta$ is a solution of the field equation for the Lagrangian density $L$.

Suppose that $\eta$ is a solution of the field equation for the Lagrangian density $L$. Then $L(\eta)=0$ and formula (31) implies that either $L_{+}(\eta)=0$ or $L_{-}(\eta)=0$; note that in view of (32) we cannot have simultaneously $L_{+}(\eta)=0$ and $L_{-}(\eta)=0$. Assume for definiteness that $L_{+}(\eta)=0$. [The case when $L_{-}(\eta)=0$ is handled similarly.] Varying $\eta$ and repeating the argument from the previous paragraph we arrive at (35). We assumed that $\eta$ is a solution of the field equation for the Lagrangian density $L$ so $\delta \int L(\eta)=0$ and formula (35) implies that $\delta \int L_{+}(\eta)=0$. As the latter is true for an arbitrary variation of $\eta$ this means that $\eta$ is a solution of the field equation for the Lagrangian density $L_{+}$.

\section{PLANE WAVE SOLUTIONS}

Suppose that $M=\mathbb{R}^{3}$ is Euclidean 3-space equipped with Cartesian coordinates $x=\left(x^{1}, x^{2}, x^{3}\right)$ and standard Euclidean metric (B9). In this section we construct a special class of explicit solutions of the field equations for our Lagrangian density (19). This construction is presented in the language of spinors.

Let us choose Pauli matrices (B10) and seek solutions of the form

$$
\xi\left(x^{0}, x^{1}, x^{2}, x^{3}\right)=e^{-i\left(p_{0} x^{0}+p \cdot x\right)} \zeta
$$

where $p_{0}$ is a real number as in formulas (3) and (4), $p=\left(p_{1}, p_{2}, p_{3}\right)$ is a real constant covector and $\zeta \neq 0$ is a constant spinor. We shall call solutions of the type (36) plane wave. In seeking plane wave solutions what we are doing is separating out all the variables, namely, the time variable $x^{0}$ and the spatial variables $x=\left(x^{1}, x^{2}, x^{3}\right)$.

Our dynamic field equation (21) is highly nonlinear so it is not a priori clear that one can seek solutions in the form of plane waves. However, plane wave solutions (36) are a special case of stationary solutions (3) and these have already been analyzed in preceding sections. 
Namely, Theorem 1 gives us an algorithm for the calculation of all plane wave solutions (36) by reducing the problem to a pair of stationary Weyl equations (5) for the time-independent spinor field

$$
\eta\left(x^{1}, x^{2}, x^{3}\right)=e^{-i p \cdot x} \zeta .
$$

Substituting formulas (B2), (B10) and (37) into equation (5) we get

$$
\left(\begin{array}{cc}
\mp p_{0}+p_{3} & p_{1}-i p_{2} \\
p_{1}+i p_{2} & \mp p_{0}-p_{3}
\end{array}\right)\left(\begin{array}{l}
\zeta^{1} \\
\zeta^{2}
\end{array}\right)=0
$$

The determinant of the matrix in the LHS of equation (38) is $p_{0}^{2}-p_{1}^{2}-p_{2}^{2}-p_{3}^{2}$ so this system has a nontrivial solution $\zeta$ if and only if $p_{0}^{2}-p_{1}^{2}-p_{2}^{2}-p_{3}^{2}=0$. Our model is invariant under rotations of the Cartesian coordinate system (orthogonal transformations of the coordinate system preserving orientation) so without loss of generality we can assume that

$$
p_{1}=p_{2}=0, \quad p_{3}= \pm p_{0}
$$

where the \pm sign is chosen to agree with that in equation (38), i.e. upper sign in (39) corresponds to upper sign in (38) and same for lower signs. Substituting formulas (39) into equation (38) and recalling our assumption (4) we conclude that, up to scaling by a nonzero complex factor, we have

$$
\zeta^{d}=\left(\begin{array}{l}
1 \\
0
\end{array}\right)
$$

Combining formulas (36), (39) and (40) we conclude that for each real $p_{0} \neq 0$ our model admits, up to a rotation of the coordinate system and complex scaling, two plane wave solutions and that these plane wave solutions are given by the explicit formula

$$
\xi^{d}=\left(\begin{array}{l}
1 \\
0
\end{array}\right) e^{-i p_{0}\left(x^{0} \pm x^{3}\right)} .
$$

Let us now rewrite the plane wave solutions (41) in terms of our original dynamical variables, coframe $\vartheta$ and density $\rho$. Substituting formulas (B2), (B10) and (41) into formulas (C1) - (C4) we get $\rho=1$ and

$$
\begin{aligned}
\vartheta^{1}{ }_{\alpha} & =\left(\begin{array}{c}
\cos 2 p_{0}\left(x^{0} \pm x^{3}\right) \\
\sin 2 p_{0}\left(x^{0} \pm x^{3}\right) \\
0
\end{array}\right) \\
\vartheta^{2}{ }_{\alpha} & =\left(\begin{array}{c}
-\sin 2 p_{0}\left(x^{0} \pm x^{3}\right) \\
\cos 2 p_{0}\left(x^{0} \pm x^{3}\right) \\
0
\end{array}\right), \quad \vartheta^{3}{ }_{\alpha}=\left(\begin{array}{l}
0 \\
0 \\
1
\end{array}\right) .
\end{aligned}
$$

Note that scaling the spinor $\zeta$ by a nonzero complex factor is equivalent to scaling the density $\rho$ by a positive real factor and time shift $x^{0} \mapsto x^{0}+$ const.

We will now establish how many different (ones that cannot be continuously transformed into one another) plane wave solutions we have. To this end, we rewrite formula (42) in the form

$$
\begin{aligned}
\vartheta^{1}{ }_{\alpha} & =\left(\begin{array}{c}
\cos 2\left|p_{0}\right|\left(x^{0}+b x^{3}\right) \\
a \sin 2\left|p_{0}\right|\left(x^{0}+b x^{3}\right) \\
0
\end{array}\right), \\
\vartheta^{2}{ }_{\alpha} & =\left(\begin{array}{c}
-a \sin 2\left|p_{0}\right|\left(x^{0}+b x^{3}\right) \\
\cos 2\left|p_{0}\right|\left(x^{0}+b x^{3}\right) \\
0
\end{array}\right), \quad \vartheta^{3}{ }_{\alpha}=\left(\begin{array}{l}
0 \\
0 \\
1
\end{array}\right)
\end{aligned}
$$

where $a$ and $b$ can, independently, take values \pm 1 . It may seem that we have a total of 4 different plane wave solutions. Recall, however, that we can perform rigid rotations of the coframe and that we have agreed (see Remark 1 at the end of Section II) to view coframes that differ by a rigid rotation as equivalent. Let us perform a rotation of the coordinate system

$$
\left(\begin{array}{c}
x^{1} \\
x^{2} \\
x^{3}
\end{array}\right) \mapsto\left(\begin{array}{c}
x^{2} \\
x^{1} \\
-x^{3}
\end{array}\right)
$$

simultaneously with a rigid rotation of the coframe

$$
\left(\begin{array}{l}
\vartheta^{1} \\
\vartheta^{2} \\
\vartheta^{3}
\end{array}\right) \mapsto\left(\begin{array}{c}
\vartheta^{2} \\
\vartheta^{1} \\
-\vartheta^{3}
\end{array}\right)
$$

It is easy to see that the above transformations turn a solution of the form (43) into a solution of this form again only with

$$
a \mapsto-a, \quad b \mapsto-b .
$$

Thus, the numbers $a$ and $b$ on their own do not characterize different plane wave solutions. Different plane wave solutions are characterized by the number $c:=a b$ which can take two values, +1 and -1 .

We have established that for a given positive frequency $\left|p_{0}\right|$ we have two essentially different types of plane wave solutions. These can be written, for example, as

$$
\begin{aligned}
\vartheta^{1}{ }_{\alpha} & =\left(\begin{array}{c}
\cos 2\left|p_{0}\right|\left(x^{0}+x^{3}\right) \\
\pm \sin 2\left|p_{0}\right|\left(x^{0}+x^{3}\right) \\
0
\end{array}\right), \\
\vartheta^{2}{ }_{\alpha} & =\left(\begin{array}{c}
\mp \sin 2\left|p_{0}\right|\left(x^{0}+x^{3}\right) \\
\cos 2\left|p_{0}\right|\left(x^{0}+x^{3}\right) \\
0
\end{array}\right), \quad \vartheta^{3}{ }_{\alpha}=\left(\begin{array}{l}
0 \\
0 \\
1
\end{array}\right) .
\end{aligned}
$$

The plane wave solutions (44) describe traveling waves of rotations. Both waves travel with the same velocity (speed of light) in the negative $x^{3}$-direction. The difference between the two solutions is in the direction of rotation of the coframe: if we fix the spatial coordinate $x^{3}$ and look at the evolution of (44) as a function of time $x^{0}$ or if we fix time $x^{0}$ and look at the evolution of (44) as a function of the spatial coordinate $x^{3}$ then one solution describes a clockwise rotation whereas the other solution 
describes an anticlockwise rotation. We identify one of the solutions (44) with a left-handed massless neutrino and the other with a right-handed massless antineutrino.

The bottom line is that our model gives the correct number, two, of distinct plane wave solutions.

\section{RELATIVISTIC REPRESENTATION OF OUR LAGRANGIAN}

In this section we work on the 4 -manifold $\mathbb{R} \times M$ equipped with Lorentzian metric (1). This manifold is an extension of the original 3-manifold $M$. We use bold type for extended quantities.

We extend our coframe as

$$
\begin{gathered}
\boldsymbol{\vartheta}^{0}{ }_{\boldsymbol{\alpha}}=\left(\begin{array}{c}
1 \\
0_{\alpha}
\end{array}\right), \\
\boldsymbol{\vartheta}_{\boldsymbol{\alpha}}^{j}=\left(\begin{array}{c}
0 \\
\vartheta^{j}{ }_{\alpha}
\end{array}\right), \quad j=1,2,3,
\end{gathered}
$$

where the bold tensor index $\boldsymbol{\alpha}$ runs through the values 0 , $1,2,3$, whereas its non-bold counterpart $\alpha$ runs through the values $1,2,3$. In particular, the $0_{\alpha}$ in formula (45) stands for a column of three zeros.

Throughout this section our original 3-dimensional coframe $\vartheta$ is allowed to depend on time $x^{0}$ in an arbitrary (not necessarily harmonic) manner, as long as the kinematic constraint (6) is maintained. Thus, our only restriction on the choice of extended 4-dimensional coframe $\boldsymbol{\vartheta}$ is formula (45) which says that the zeroth element of the coframe is prescribed as the conormal to the original Riemannian 3-manifold $M$.

The extended metric (11) is expressed via the extended coframe (45) and (46) as

$$
\mathrm{g}=\mathbf{o}_{\mathrm{jk}} \boldsymbol{\vartheta}^{\mathrm{j}} \otimes \boldsymbol{\vartheta}^{\mathrm{k}}
$$

where $\mathbf{o}_{\mathbf{j k}}=\mathbf{o}^{\mathbf{j k}}:=\operatorname{diag}(-1,+1,+1,+1)$ (compare with formula (6) $)$. The extended axial torsion is

$$
\begin{aligned}
& \mathbf{T}^{\mathrm{ax}}:=\frac{1}{3} \mathbf{o}_{\mathbf{j k}} \boldsymbol{\vartheta}^{\mathbf{j}} \wedge d \boldsymbol{\vartheta}^{\mathbf{k}} \\
= & \frac{1}{3}(-\underbrace{\boldsymbol{\vartheta}^{0} \wedge d \boldsymbol{\vartheta}^{0}}_{=0}+\boldsymbol{\vartheta}^{1} \wedge d \boldsymbol{\vartheta}^{1}+\boldsymbol{\vartheta}^{2} \wedge d \boldsymbol{\vartheta}^{2}+\boldsymbol{\vartheta}^{3} \wedge d \boldsymbol{\vartheta}^{3})
\end{aligned}
$$

where $d$ denotes the exterior derivative on $\mathbb{R} \times M$ (compare with formula (8)). Formula (48) can be rewritten as

$$
\mathbf{T}^{\mathrm{ax}}=T^{\mathrm{ax}}-\boldsymbol{\vartheta}^{0} \wedge \dot{\vartheta}
$$

with $T^{\text {ax }}$ and $\dot{\vartheta}$ defined by formulas (8) and (15) respectively. Squaring (49) we get $\left\|\mathbf{T}^{\mathrm{ax}}\right\|^{2}=\left\|T^{\mathrm{ax}}\right\|^{2}-\|\dot{\vartheta}\|^{2}$ which implies that our Lagrangian density (19) can be rewritten as

$$
L(\vartheta, \rho)=\left\|\mathbf{T}^{\mathrm{ax}}\right\|^{2} \rho .
$$

The point of the arguments presented in this section was to show that if one adopts the relativistic point of view then our Lagrangian density (19) takes the especially simple form (50). Formula (50) is also useful in that it allows us to see that our Lagrangian density is invariant under conformal rescalings of the 4-dimensional Lorentzian metric g: the arguments from Section II (see formulas (9)-(11) and (13)) carry over to the 4dimensional setting without change.

A consistent pursuit of the relativistic approach would require the variation of all four elements of the extended coframe, giving three extra dynamical degrees of freedom (Lorentz boosts in three directions). We do not do this in the current paper, assuming instead that the zeroth element of the extended coframe is specified by formula (45).

\section{DISCUSSION}

The mathematical model presented in Section [I is, effectively, a special case of the theory of teleparallelism 2-4]. Modern reviews of teleparallelism can be found in [7, 10 14]. The differences between our mathematical model and those commonly used in teleparallelism are as follows.

- We assume the metric to be prescribed (fixed) whereas in teleparallelism it is traditional to view the metric as a dynamical variable. In other words, in teleparallelism it is customary to view (6) not as a kinematic constraint but as a definition of the metric and, consequently, to vary the coframe without any constraints. This is not surprising as most, if not all, authors who contributed to teleparallelism came to the subject from General Relativity.

- We take the density of our continuum $\rho$ to be a dynamical variable whereas in teleparallelism the tradition is to prescribe it as $\rho=\sqrt{\operatorname{det} g}$. Taking $\rho$ to be a dynamical variable is, of course, equivalent to introducing an extra real positive scalar field $\rho / \sqrt{\operatorname{det} g}$ into our model

- We choose a very particular Lagrangian density (50) containing only one irreducible piece of torsion (axial) whereas in teleparallelism it is traditional to choose a more general Lagrangian containing all three pieces (axial, vector and tensor): see formula (26) in [7]. In choosing our particular Lagrangian density (50) we were guided by the principles of conformal invariance, simplicity and analogy with Maxwell's theory.

The main result of our paper is Theorem 1 which establishes that in the stationary setting (prescribed harmonic oscillation in time) our mathematical model is equivalent to a pair of massless Weyl equations (2). The advantage of our approach is that it makes the Weyl equation look natural to someone with a continuum mechanics background. The downside is that our mathematical model 
is nonlinear which makes it look unnatural to someone with a quantum mechanical background.

The situation here has a certain similarity with integrable systems. Say, the Korteweg-de Vries equation (mathematical model of waves on shallow water surfaces) is nonlinear but the inverse scattering transform reduces it to the analysis of a spectral problem for a linear SturmLiouville operator. In our paper we go the other way round, reformulating the spectral problem for the linear Weyl operator as a nonlinear equation from continuum mechanics.

From a purely mathematical viewpoint Theorem [1 is unusual in that it states that a (particular) second order partial differential equation is equivalent to a pair of first order partial differential equations, which is actually hard to believe. Indeed, let us choose a 2-dimensional hypersurface $S$ on the 3-manifold $M$ and set a Cauchy problem on this surface. When dealing with a second order partial differential equation one expects to be able to prescribe the value of the spinor field $\eta$ on the surface $S$ as well as its normal derivative, whereas when dealing with a first order partial differential equation one expects to be able to prescribe the value of the spinor field $\eta$ only (the value of the normal derivative of $\eta$ on the surface $S$ will be determined by the equation). This argument appears to show that there is no way a second order partial differential equation can be reduced to a pair of first order equations. However, our second order partial differential equation happens to be degenerate and does not admit the setting of a standard Cauchy problem. This degeneracy manifests itself in the property of scaling covariance of our stationary Lagrangian density (28), see Section VI for details. Scaling covariance implies that our stationary Lagrangian density (28) vanishes on solutions of the (second order) field equation which means that the value of the spinor field $\eta$ on the surface $S$ and its normal derivative cannot be chosen independently. In order to allay fears that there is something inherently wrong with our construction we provide in Appendix E an elementary example showing by means of an explicit calculation that a second order differential equation with Lagrangian of the form (31) and (33) does indeed reduce to a pair of first order equations.

Our construction exhibits a certain similarity with the Riccati equation. Recall that the Riccati equation is a nonlinear first order differential equation which reduces to a linear second order differential equation. We go the other way round, reducing a nonlinear second order equation to a pair of linear first order equations. However, unlike the Riccati equation, our construction works not only for ordinary differential equations but also for partial differential equations.

Theorem 1 leaves us with two issues unresolved.

A What can be said about the general case, when the spinor field $\xi$ is an arbitrary function of all spacetime coordinates $\left(x^{0}, x^{1}, x^{2}, x^{3}\right)$ and is not necessarily of the form (3)?
B What can be said about the relativistic version of our model described in Section VIII?

The two issues are, of course, related: both arise because in formulating our basic model in Section [II we adopted the Newtonian approach which specifies the time coordinate $x^{0}$ ("absolute time").

We plan to tackle issue A by means of perturbation theory. Namely, assuming the metric to be flat (as in Section VII), we start with a plane wave (36) and then seek the unknown spinor field $\xi$ in the form

$$
\xi\left(x^{0}, x^{1}, x^{2}, x^{3}\right)=e^{-i\left(p_{0} x^{0}+p \cdot x\right)} \zeta\left(x^{0}, x^{1}, x^{2}, x^{3}\right)
$$

where $\zeta$ is a slowly varying spinor field. Here "slowly varying" means that second derivatives of $\zeta$ can be neglected compared to the first. Our conjecture is that the application of a formal perturbation argument will yield the Weyl equation (2) for the spinor field $\xi$.

We plan to tackle issue B by means of perturbation theory as well. The relativistic version of our model has three extra field equations corresponding to the three extra dynamical degrees of freedom (Lorentz boosts in three directions). Our conjecture is that if we take a solution of the nonrelativistic problem which is a perturbation of a plane wave (as in the previous paragraph) then, at a perturbative level, this solution will automatically satisfy the three extra field equations. In other words, we conjecture that our nonrelativistic model possesses relativistic invariance at the perturbative level.

The detailed analysis of the two issues flagged up above will be the subject of a separate paper.

\section{Appendix A: General notation}

Our general notation mostly follows [5, 15], the only major difference being that we changed the signature of Lorentzian metric $\mathbf{g}_{\boldsymbol{\alpha} \boldsymbol{\beta}}$ from +--- to -+++ . The latter is more natural when promoting the Newtonian continuum mechanics approach.

We use Greek letters for tensor (holonomic) indices and Latin letters for frame (anholonomic) indices.

We identify differential forms with covariant antisymmetric tensors. Given a pair of real covariant antisymmetric tensors $P$ and $Q$ of rank $r$ we define their dot product as $P \cdot Q:=\frac{1}{r !} P_{\alpha_{1} \ldots \alpha_{r}} Q_{\beta_{1} \ldots \beta_{r}} g^{\alpha_{1} \beta_{1}} \ldots g^{\alpha_{r} \beta_{r}}$. We also define $\|P\|^{2}:=P \cdot P$.

All our constructions are local and occur in a neighborhood of a given point $P$ of the 3 -manifold $M$. We allow only changes of local coordinates $x^{\alpha}, \alpha=1,2,3$, which preserve orientation.

Working in local coordinates with specified orientation allows us to define the Hodge star: we define the action of $*$ on a rank $r$ antisymmetric tensor $R$ as

$$
(* R)_{\alpha_{r+1} \ldots \alpha_{3}}:=(r !)^{-1} \sqrt{\operatorname{det} g} R^{\alpha_{1} \ldots \alpha_{r}} \varepsilon_{\alpha_{1} \ldots \alpha_{3}}
$$

where $\varepsilon$ is the totally antisymmetric quantity, $\varepsilon_{123}:=+1$. 
Coframes $\vartheta$ fall into two separate categories, depending on the sign of $\operatorname{det} \vartheta_{\alpha}^{j}$. We choose to work with coframes satisfying the condition

$$
\operatorname{det} \vartheta_{\alpha}^{j}>0
$$

Condition (A2) means that orientation encoded in our coframe agrees with that encoded in our coordinate system.

An orthogonal transformation of a coframe is a linear map

$$
\vartheta^{j} \mapsto \tilde{\vartheta}^{j}=O_{k}^{j} \vartheta^{k}
$$

where the $O^{j}{ }_{k}$ are real scalar functions satisfying the condition $\delta_{j i} O^{j}{ }_{k} O^{i}{ }_{r}=\delta_{k r}$. Of course, orthogonal transformations map coframes into coframes, i.e. they preserve the kinematic constraint (6). We call an orthogonal transformation special (or a rotation) if the $O^{j}{ }_{k}$ satisfy the additional condition $\operatorname{det} O^{j}{ }_{k}=+1$. Any two coframes satisfying condition (A2) are related by a special orthogonal transformation (rotation).

\section{Appendix B: Spinor notation}

Our spinor notation mostly follows [16], the difference being that we changed the signature of Lorentzian metric.

We use two-component complex-valued spinors (Weyl spinors) whose indices run through the values 1,2 or $\dot{1}, \dot{2}$. Complex conjugation makes the undotted indices dotted and vice versa.

Define the "metric spinor"

$$
\epsilon_{a b}=\epsilon_{\dot{a} \dot{b}}=\epsilon^{a b}=\epsilon^{\dot{a} \dot{b}}=\left(\begin{array}{cc}
0 & -1 \\
1 & 0
\end{array}\right)
$$

with the first index enumerating rows and the second enumerating columns. We will be using the spinor (B1) for lowering and raising spinor indices.

We define

$$
\sigma_{0 \dot{a} b}=\sigma_{0}{ }^{\dot{a} b}=\left(\begin{array}{cc}
1 & 0 \\
0 & 1
\end{array}\right), \sigma_{\dot{a} b}^{0}=\sigma^{0 \dot{a} b}=-\left(\begin{array}{cc}
1 & 0 \\
0 & 1
\end{array}\right) .
$$

The spinor (B2) can also be used for raising and lowering spinor indices. This is a feature of the nonrelativistic setting, when we have a specified time coordinate $t=x^{0}$ and transformations of spatial local coordinates $x^{\alpha}, \alpha=$ $1,2,3$, do not involve time.

Let $\mathfrak{v}$ be the real vector space of trace-free Hermitian $2 \times 2$ matrices $\sigma_{\dot{a} b}$. Pauli matrices $\sigma_{\alpha \dot{a} b}, \alpha=1,2,3$, are a basis in $\mathfrak{v}$ satisfying

$$
\sigma_{\alpha \dot{a} b} \sigma_{\beta}^{\dot{a} c}+\sigma_{\beta \dot{a} b} \sigma_{\alpha}^{\dot{a} c}=-2 g_{\alpha \beta} \delta_{b}^{c}
$$

where $\sigma_{\beta}{ }^{\dot{a} c}:=\epsilon^{\dot{a} \dot{e}} \sigma_{\beta \dot{e} d} \epsilon^{c d}$. Note that formula (B3) automatically implies an analogous formula for the extended metric (1):

$$
\sigma_{\boldsymbol{\alpha} \dot{a} b} \sigma_{\boldsymbol{\beta}}^{\dot{a} c}+\sigma_{\boldsymbol{\beta} \dot{a} b} \sigma_{\boldsymbol{\alpha}}^{\dot{a} c}=-2 \mathbf{g}_{\boldsymbol{\alpha} \boldsymbol{\beta}} \delta_{b}^{c}
$$

where the bold tensor indices $\boldsymbol{\alpha}, \boldsymbol{\beta}$ run through the values $0,1,2,3$.

Of course, our Pauli matrices $\sigma_{\alpha}, \alpha=1,2,3$, are not uniquely defined: if $\sigma_{\alpha}=\sigma_{\alpha \dot{a} b}$ are Pauli matrices then so are the matrices $U^{*} \sigma_{\alpha} U$ where $U$ is an arbitrary special (det $U=1$ ) unitary matrix-function. Note also that under coordinate transformations our Pauli matrices $\sigma_{\alpha a b}$ transform as components of a covector: this is indicated by the Greek subscript $\alpha$.

Let us mention a useful identity for Pauli matrices, very similar to (B4) but with contraction over tensor indices instead of spinor ones:

$$
\sigma_{\boldsymbol{\alpha} \dot{a} b} \sigma_{\dot{c} d}^{\boldsymbol{\alpha}}=-2 \epsilon_{\dot{a} \dot{c}} \epsilon_{b d}
$$

We define the covariant derivatives of spinor fields as

$$
\begin{array}{ll}
\nabla_{\mu} \xi^{a}=\partial_{\mu} \xi^{a}+\Gamma_{\mu b}^{a} \xi^{b}, & \nabla_{\mu} \xi_{a}=\partial_{\mu} \xi_{a}-\Gamma_{\mu a}^{b} \xi_{b} \\
\nabla_{\mu} \eta^{\dot{a}}=\partial_{\mu} \eta^{\dot{a}}+\bar{\Gamma}_{\mu \dot{b}}^{\dot{a}} \eta^{\dot{b}}, & \nabla_{\mu} \eta_{\dot{a}}=\partial_{\mu} \eta_{\dot{a}}-\bar{\Gamma}_{\mu \dot{a}}^{\dot{b}} \eta_{\dot{b}}
\end{array}
$$

where $\bar{\Gamma}_{\mu \dot{b}}^{\dot{a}}=\overline{\Gamma_{\mu b}^{a}}$ and $\mu$ runs through the values $1,2,3$. The explicit formula for the spinor connection coefficients $\Gamma_{\mu b}^{a}$ can be derived from the following two conditions:

$$
\begin{gathered}
\nabla_{\mu} \epsilon_{a b}=0, \\
\nabla_{\mu} \sigma_{\dot{a} b}^{\alpha}=0,
\end{gathered}
$$

where

$$
\nabla_{\mu} \sigma_{\dot{a} b}^{\alpha}=\partial_{\mu} \sigma_{\dot{a} b}^{\alpha}+\Gamma_{\mu \beta}^{\alpha} \sigma_{\dot{a} b}^{\beta}-\bar{\Gamma}_{\mu \dot{a}}^{\dot{c}} \sigma_{\dot{c} b}^{\alpha}-\Gamma_{\mu b}^{d} \sigma_{\dot{a} d}^{\alpha}
$$

and $\Gamma_{\alpha \gamma}^{\beta}=\left\{\begin{array}{c}\beta \\ \alpha \gamma\end{array}\right\}:=\frac{1}{2} g^{\beta \delta}\left(\partial_{\alpha} g_{\gamma \delta}+\partial_{\gamma} g_{\alpha \delta}-\partial_{\delta} g_{\alpha \gamma}\right)$ are the Christoffel symbols. Conditions (B6), (B7) give an overdetermined system of linear algebraic equations for $\operatorname{Re} \Gamma_{\mu b}^{a}, \operatorname{Im} \Gamma_{\mu b}^{a}$ the unique solution of which is

$$
\Gamma_{\mu b}^{a}=-\frac{1}{4} \sigma_{\alpha}^{\dot{c} a}\left(\partial_{\mu} \sigma_{\dot{c} b}^{\alpha}+\Gamma_{\mu \beta}^{\alpha} \sigma_{\dot{c} b}^{\beta}\right) .
$$

Observe that the sign in the RHS of formula (B8) is different from that of formula (A.9) in [16]. This is because we changed the signature of Lorentzian metric.

Note that for the standard Euclidean metric

$$
g_{\alpha \beta}=\operatorname{diag}(1,1,1)
$$

the traditional choice of Pauli matrices is

$$
\sigma_{1 \dot{a} b}=\left(\begin{array}{cc}
0 & 1 \\
1 & 0
\end{array}\right), \sigma_{2 \dot{a} b}=\left(\begin{array}{cc}
0 & -i \\
i & 0
\end{array}\right), \sigma_{3 \dot{a} b}=\left(\begin{array}{cc}
1 & 0 \\
0 & -1
\end{array}\right) \text {. }
$$

\section{Appendix C: Correspondence between coframes and spinors}

In dimension 3 a coframe $\vartheta$ and a (positive) density $\rho$ are equivalent to a nonvanishing spinor field $\xi$ modulo the sign of $\xi$ in accordance with the formulas

$$
s=\bar{\xi}^{\dot{a}} \sigma_{0 \dot{a} b} \xi^{b},
$$




$$
\begin{gathered}
\rho=s \sqrt{\operatorname{det} g} \\
\left(\vartheta^{1}+i \vartheta^{2}\right)_{\alpha}=s^{-1} \epsilon^{\dot{c} \dot{b}} \sigma_{0 \dot{b} a} \xi^{a} \sigma_{\alpha \dot{c} d} \xi^{d} \\
\vartheta_{\alpha}^{3}=s^{-1} \bar{\xi}^{\dot{a}} \sigma_{\alpha \dot{a} b} \xi^{b} .
\end{gathered}
$$

The above formulas are a special case of those from [17].

We assume that our Pauli matrices are chosen in such a way that the coframe $\vartheta$ defined by formulas (C1), C3 and (C4) satisfies condition (A2) for all $\xi \neq 0$. Of course, the sign of $\operatorname{det} \vartheta^{j}{ }_{\alpha}$ can always be changed by switching from original Pauli matrices to their complex conjugates.

Note that if we have the standard Euclidean metric (B9), use traditional Pauli matrices (B10) and take

$$
\xi^{a}=\left(\begin{array}{l}
1 \\
0
\end{array}\right)
$$

then formulas (C1), (C3) and (C4) give us

$$
\vartheta_{\alpha}^{j}=\delta_{\alpha}^{j}
$$

\section{Appendix D: Spinor representation of axial torsion and angular velocity}

We show in this appendix that the Hodge dual of axial torsion (8) is expressed via the spinor field $\xi$ as

$$
* T^{\mathrm{ax}}=-\frac{2 i\left(\bar{\xi}^{\dot{a}} \sigma^{\alpha}{ }_{a b} \nabla_{\alpha} \xi^{b}-\xi^{b} \sigma^{\alpha}{ }_{\dot{a} b} \nabla_{\alpha} \bar{\xi}^{\dot{a}}\right)}{3 \bar{\xi}^{\dot{c}} \sigma_{0 \dot{c} d} \xi^{d}}
$$

and that the vector of angular velocity $\omega$ defined by formula (17) is expressed via the spinor field $\xi$ as

$$
\omega_{\alpha}=\frac{i\left(\bar{\xi}^{\dot{a}} \sigma_{\alpha \dot{a} b} \partial_{0} \xi^{b}-\xi^{b} \sigma_{\alpha \dot{a} b} \partial_{0} \bar{\xi}^{\dot{a}}\right)}{\bar{\xi}^{\dot{c}} \sigma_{0 \dot{c} d} \xi^{d}} .
$$

Note that formulas (D1) and (D2) are invariant under the rescaling of our spinor field by an arbitrary nonvanishing real scalar function.

Formulas (D1) and (D2) are proved by direct substitution of formulas (C1), (C3) and (C4) into (8) and (17) respectively. In order to simplify calculations we observe that the expressions in the left- and right-hand sides of formulas (D1) and (D2) have an invariant nature, hence it is sufficient to prove these formulas for standard Euclidean metric (B9), traditional Pauli matrices (B10) and at a point at which the spinor field takes the value (C5).

We have

$$
\begin{aligned}
\xi^{a} & =\left(\begin{array}{c}
1+\delta \xi^{1} \\
\delta \xi^{2}
\end{array}\right), \\
\left(\vartheta^{1}+i \vartheta^{2}\right)_{\alpha} & =\left(\begin{array}{c}
1+\delta \xi^{1}-\delta \bar{\xi}^{i} \\
i+i \delta \xi^{1}-i \delta \bar{\xi}^{1} \\
-2 \delta \xi^{2}
\end{array}\right),
\end{aligned}
$$

$$
\begin{gathered}
\vartheta_{\alpha}^{3}=\left(\begin{array}{c}
\delta \xi^{2}+\delta \bar{\xi}^{2} \\
-i \delta \xi^{2}+i \delta \bar{\xi}^{\dot{2}} \\
1
\end{array}\right) \\
{\left[\operatorname{curl}\left(\vartheta^{1}+i \vartheta^{2}\right)\right]_{\alpha}=\left(\begin{array}{c}
-2 \nabla_{2} \xi^{2}-\nabla_{3}\left(i \xi^{1}-i \bar{\xi}^{\dot{1}}\right) \\
2 \nabla_{1} \xi^{2}+\nabla_{3}\left(\xi^{1}-\bar{\xi}^{\dot{1}}\right) \\
\nabla_{1}\left(i \xi^{1}-i \bar{\xi}^{\dot{1}}\right)-\nabla_{2}\left(\xi^{1}-\bar{\xi}^{\dot{1}}\right)
\end{array}\right)} \\
{\left[\operatorname{curl} \vartheta^{3}\right]_{\alpha}=\left(\begin{array}{c}
-\nabla_{3}\left(-i \xi^{2}+i \dot{\xi}^{\dot{2}}\right) \\
\nabla_{3}\left(\xi^{2}+\bar{\xi}^{2}\right) \\
\nabla_{1}\left(-i \xi^{2}+i \bar{\xi}^{2}\right)-\nabla_{2}\left(\xi^{2}+\bar{\xi}^{\dot{2}}\right)
\end{array}\right)} \\
{\left[\partial_{0}\left(\vartheta^{1}+i \vartheta^{2}\right)\right]_{\alpha}=\left(\begin{array}{c}
\partial_{0} \xi^{1}-\partial_{0} \bar{\xi}^{\dot{1}} \\
i \partial_{0} \xi^{1}-i \partial_{0} \bar{\xi}^{1} \\
-2 \partial_{0} \xi^{2}
\end{array}\right)}
\end{gathered}
$$$$
\left[\partial_{0} \vartheta^{3}\right]_{\alpha}=\left(\begin{array}{c}
\partial_{0} \xi^{2}+\partial_{0} \bar{\xi}^{\dot{2}} \\
-i \partial_{0} \xi^{2}+i \partial_{0} \bar{\xi}^{\dot{2}} \\
0
\end{array}\right)
$$

where curl $u:=* d u$.

We rewrite the formulas for $* T^{\mathrm{ax}}$ and $\omega$ in the form

$$
\begin{aligned}
& * T^{\mathrm{ax}}=\frac{1}{6}\left(\vartheta^{1}-i \vartheta^{2}\right) \cdot \operatorname{curl}\left(\vartheta^{1}+i \vartheta^{2}\right) \\
& +\frac{1}{6}\left(\vartheta^{1}+i \vartheta^{2}\right) \cdot \operatorname{curl}\left(\vartheta^{1}-i \vartheta^{2}\right)+\frac{1}{3} \vartheta^{3} \cdot \operatorname{curl} \vartheta^{3} \\
& \omega=\frac{1}{4}\left(\vartheta^{1}-i \vartheta^{2}\right) \times \partial_{0}\left(\vartheta^{1}+i \vartheta^{2}\right) \\
& \quad+\frac{1}{4}\left(\vartheta^{1}+i \vartheta^{2}\right) \times \partial_{0}\left(\vartheta^{1}-i \vartheta^{2}\right)+\frac{1}{2} \vartheta^{3} \times \partial_{0} \vartheta^{3}
\end{aligned}
$$

where $u \cdot v:=u_{\alpha} v^{\alpha}$ (note the absence of complex conjugation) and $u \times v:=*(u \wedge v)$. Substituting formulas (C6), (D3) and (D4) into formula (D7) we get

$* T^{\mathrm{ax}}=-\frac{2 i}{3}\left[\nabla_{3} \xi^{1}+\left(\nabla_{1}-i \nabla_{2}\right) \xi^{2}-\nabla_{3} \bar{\xi}^{\dot{1}}-\left(\nabla_{1}+i \nabla_{2}\right) \dot{\xi}^{2}\right]$

which coincides with the RHS of formula (D1). Substituting formulas (C6), (D5) and (D6) into formula (D8) we get

$$
\omega_{\alpha}=i\left(\begin{array}{c}
\partial_{0} \xi^{2}-\partial_{0} \bar{\xi}^{\dot{2}} \\
-i \partial_{0} \xi^{2}-i \partial_{0} \bar{\xi}^{\dot{2}} \\
\partial_{0} \xi^{1}-\partial_{0} \bar{\xi}^{\dot{1}}
\end{array}\right)
$$

which coincides with the RHS of formula (D2).

An alternative way of proving formulas of the type (D1) and (D2) is to choose Pauli matrices $\sigma_{\boldsymbol{\alpha}}, \boldsymbol{\alpha}=$ $0,1,2,3$, in such a way that a given nonvanishing spinor field $\xi$ takes the value (C5) in some neighborhood of a given point (as opposed to only the point itself). This approach was adopted, for example, in [5, 18 20]. 


\section{Appendix E: Toy model}

In this appendix we present a toy model showing that a second order differential equation with Lagrangian of the form (31) and (33) reduces to a pair of first order equations.

We work on the real line $\mathbb{R}$ parametrized by the coordinate $x$. The dynamical variable (unknown quantity) is the scalar function $\eta: \mathbb{R} \rightarrow \mathbb{C} \backslash\{0\}$. Differentiation in $x$ is denoted by $\nabla$.

Consider a pair of first order linear ordinary differential equations

$$
i \nabla \eta \pm \eta=0
$$

The corresponding Lagrangians are

$$
L_{ \pm}(\eta):=\frac{i}{2}(\bar{\eta} \nabla \eta-\eta \nabla \bar{\eta}) \pm|\eta|^{2} .
$$

Equations (E1) are simplified versions of the stationary Weyl equations (5) and Lagrangians (E2) are simplified versions of the stationary Weyl Lagrangians (29). Note that the Lagrangians (E2) possess the property of scaling covariance (33) where $h: \mathbb{R} \rightarrow \mathbb{R}$ is an arbitrary scalar function.

By analogy with (31), put

$$
L(\eta):=\frac{2 L_{+}(\eta) L_{-}(\eta)}{L_{+}(\eta)-L_{-}(\eta)}=\left[\frac{i(\bar{\eta} \nabla \eta-\eta \nabla \bar{\eta})}{2|\eta|}\right]^{2}-|\eta|^{2} .
$$

The corresponding field equation (Euler-Lagrange equation) is

$$
\begin{aligned}
& i\left\{\frac{(\nabla \eta)}{|\eta|}-\frac{\eta(\bar{\eta} \nabla \eta-\eta \nabla \bar{\eta})}{2|\eta|^{3}}+\nabla \frac{\eta}{|\eta|}\right\}\left[\frac{i(\bar{\eta} \nabla \eta-\eta \nabla \bar{\eta})}{2|\eta|}\right] \\
& -\eta=0
\end{aligned}
$$

where the last $\nabla$ in the curly brackets acts on all the terms to the right, including those in the square brackets. Equation (E4) is a second order nonlinear ordinary differential equation which does not appear to bear any resemblance to the first order linear ordinary differential equations (E1).

Let us switch to the polar representation of the complex function $\eta$ :

$$
\eta=r e^{-i \varphi}
$$

where $r: \mathbb{R} \rightarrow(0,+\infty)$ and $\varphi: \mathbb{R} \rightarrow \mathbb{R}$ are the new dynamical variables (unknown quantities). Substituting formula (E5) into equation (E4) and multiplying by $e^{i \varphi}$ we arrive at the polar representation of our field equation:

$$
2 i(\nabla r)(\nabla \varphi)+r(\nabla \varphi)^{2}+i r \nabla \nabla \varphi-r=0 .
$$

Separating the real and imaginary parts we rewrite the latter as a system or real equations

$$
r(\nabla \varphi)^{2}-r=0, \quad 2(\nabla r)(\nabla \varphi)+r \nabla \nabla \varphi=0,
$$

which, in turn, is equivalent to

$$
\nabla \varphi=\mp 1, \quad \nabla r=0 .
$$

This shows that a complex function $\eta$ is a solution of equation (E4) if and only if it is a solution of one of the two equations (E1).

Of course, the explicit calculations carried out above were unnecessary because the toy model considered in this appendix is covered by the abstract argument presented in Section VI. The point of these explicit calculations was to illustrate the degeneracy of field equations for Lagrangians of the form (31) and (33): looking at (E6) one sees the absence of second derivatives.

\section{Acknowledgments}

The authors are grateful to C. G. Böhmer, F. E. A. Johnson and Yu. N. Obukhov for stimulating discussions.
[1] E. Cosserat and F. Cosserat, Théorie des corps déformables (Librairie Scientifique A. Hermann et fils, Paris, 1909), ISBN 978-1429704847, reprinted by Cornell University Library.

[2] É. Cartan and A. Einstein, Letters on absolute parallelism, 1929-1932 (Princeton University Press, Princeton, N.J., 1979), ISBN 0-691-08229-4, original text with English translation by Jules Leroy and Jim Ritter, Edited by Robert Debever.

[3] A. Unzicker and T. Case, Translation of Einstein's attempt of a unified field theory with teleparallelism (2005), URL http://arxiv .org/abs/physics/0503046.

[4] T. Sauer, Historia Math. 33, 399 (2006), ISSN 0315-0860, URL http://dx.doi.org/10.1016/j.hm.2005.11.005
[5] J. Burnett and D. Vassiliev, J. Math. Phys. 50, 102501, 17 (2009), ISSN 0022-2488, URL http://dx.doi.org/10.1063/1.3204975.

[6] L. D. Landau and E. M. Lifshitz, Course of theoretical physics. Vol. 7 (Pergamon Press, Oxford, 1986), 3rd ed., ISBN 0-08-033916-6, theory of elasticity, Translated from the Russian by J. B. Sykes and W. H. Reid.

[7] F. W. Hehl and Y. N. Obukhov, Ann. Fond. Louis de Broglie 32, 157 (2007), ISSN 0182-4295.

[8] Y. N. Obukhov, Phys. Lett. A 90, 13 (1982), ISSN 0375-9601, URL http://dx.doi.org/10.1016/0375-9601(82)90037-8.

[9] J. M. Nester, J. Math. Phys. 33, 910 (1992), ISSN 00222488, URL http://dx.doi.org/10.1063/1.529742 
[10] F. W. Hehl, J. Nitsch, and P. von der Heyde, in General relativity and gravitation, Vol. 1 (Plenum, New York, 1980), pp. 329-355.

[11] F. Gronwald and F. W. Hehl, in Quantum gravity (Erice, 1995) (World Sci. Publ., River Edge, NJ, 1996), vol. 10 of Sci. Cult. Ser. Phys., pp. 148-198.

[12] U. Muench, F. Gronwald, and F. W. Hehl, Gen. Relativity Gravitation 30, 933 (1998), ISSN 0001-7701, URL http://dx.doi.org/10.1023/A:1026616326685

[13] M. Blagojević, Gravitation and gauge symmetries, Series in High Energy Physics, Cosmology and Gravitation (IOP Publishing Ltd., Bristol, 2002), ISBN 0-7503-07676.

[14] Y. N. Obukhov and J. G. Pereira, Phys. Rev. D (3) 67, 044016, 17 (2003), ISSN 0556-2821, URL http://dx.doi.org/10.1103/PhysRevD.67.044016

[15] D. Vassiliev, Phys. Rev. D 75, 025006,
$6 \quad$ (2007), ISSN 1550-7998, http://dx.doi.org/10.1103/PhysRevD.75.025006

[16] V. Pasic and D. Vassiliev, Classical Quantum Gravity 22, 3961 (2005), ISSN 0264-9381, URL http://dx.doi.org/10.1088/0264-9381/22/19/010

[17] J. B. Griffiths and R. A. Newing, J. Phys. A 3, 269 (1970), ISSN 0305-4470.

[18] A. Dimakis and F. Müller-Hoissen, J. Math. Phys. 26, 1040 (1985), ISSN 0022-2488, URL http://dx.doi.org/10.1063/1.526535.

[19] A. Dimakis and F. Müller-Hoissen, Phys. Lett. A 142, 73 (1989), ISSN 0375-9601, URL http://dx.doi.org/10.1016/0375-9601(89)90162-X.

[20] A. Dimakis and F. Müller-Hoissen, Classical Quantum Gravity 7, 283 (1990), ISSN 0264-9381, URL http://stacks.iop.org/0264-9381/7/283 